\title{
Gait Recognition Based on Silhouettes Sequences and Neural Networks for Human Identification
}

\author{
Molhema Mohualdeen*, Magdi Baker \\ Faculty of Engineering and Technology, University of Gezira, Sudan
}

\begin{tabular}{l}
\hline \hline Article Info \\
\hline Article history: \\
Received Nov 15, 2018 \\
Revised Jan 16, 2018 \\
Accepted Jan 30, 2018 \\
\hline
\end{tabular}

\section{Keyword:}

Discrete wavelet

Gait cycle

Gait recognition

Neural network

Silhouette images

\begin{abstract}
Human gait recognition is moving ahead by the need for automated person identification and verification at a distance in many applications. In this paper our system presented silhouette videos and neural networks based system for human identification. For each sequence of silhouette images, an automated Region of Interest (ROI) algorithm applied to reduce dimensionality, extract gait features has been attempted with 3level 2Dimension Discrete Wavelet Transform (3L-2D-DWT), edges detection and gait cycle were used to extract relevant feature. Back propagations neural networks used as pattern recognition. The MATLAB software graphics user interface is designed to display result and simplify the use of the system. The developed system has been evaluated using (TUM-IITKGP) which contains three different type of walking categories and the results demonstrate that the proposed system achieves $98.8 \%, 95.87 \%$ and $88 \%$ for normal walk, walking with backpack and walking with hand in pocket category respectively of correct recognition. We concluded that the different side of view movement increase reliability of the key extracted feature and improve the neural network performance which opens a scope for further development.
\end{abstract}

Copyright (C) 2018 Institute of Advanced Engineering and Science. All rights reserved.

Corresponding Author:

Molhema Mohualdeen,

Faculty of Engineering and Technology, University of Gezira, Sudan

Email: molhema_ee@hotmail.com

\section{INTRODUCTION}

Security issues have made biometrics an important means of identification and human authentication. The methods of the recognition currently, such as fingerprint recognition, facial recognition or iris recognition require physical contact or people's cooperation, So it is difficult to identify individuals using these traditional methods, but on the other hand the unique body movement can help [1] as in a gait biometric which is a relatively new biometric identification technique designed to identify people at a distance by the way they walk without the need to a physical contact.

From a technological perspective, atrecognition can be classified into three categories, Machine Vision (MV), floor sensor and wearable sensor based. MV category is prefered because it's simple, effective in terms of cost, continuous authentication and non-intrusive [2] also there are two ways to recognize human gait based on MV model-based and model-free. Model free based deal with gait images sequences directly, on the other hand; model based need more processes and also have a high cost comparing with model free based [3]. Here a model-free approache is used which is not sensitive to the quality of silhouettes.

This paper proposes a model free approach for gait recognition. For this, silhouette videos database and neural network based system have been used with an automated Region Of Interest (ROI) algorithm to reduce dimensionality, 3level 2Dimention Discrete Wavelet Transform (3L-2D-DWT) and gait cycle for features extraction. 


\subsection{Literature Review}

Kohei Arai, Rose. [4] designed a system using model free motion based and model motion based as spatial and temporal information. (GEM) as a spatial feature and velocity as a temporal feature extraction, and classify the data using (SVM). Implemented in the (CASIA) Gait Database, with $97.47 \%$ classification accuracy when only spatial information processed and $97.63 \%$ if spatial and temporal information processed.

L.R Sudha, R. Bhavani. [5] Proposed multiple gait components, i.e., spatial, temporal and wavelet are extracted from the silhouette gait sequences and fused for the development of a high performance classification system. Temporal features gives poor performance while using separately, improves performance rate when fused with other features with $97.91 \%$ accuracy.

Jiwen $\mathrm{Lu}$, et al. [6], proposed a sparse reconstruction based metric learning approach for gait-based human identity and gender recognition from arbitrary walking directions. applied (SRML) method on the existing (USF) and (CASIA-B) gait datasets. approach was achieved 87.6\% rank-1 identity recognition rate and $93.1 \%$ gender recognition rate on (ADSC-AWD) dataset containing 20 subjects, respectively.

Jinyan Chen, Gang Li. [7], developed a difference image and two-dimensional principal component analysis based gait identification and verification method. The difference image is obtained by comparing two adjacent silhouettes. By projecting difference image to $\mathrm{X}$-axis and Y-axis they got feature matrix. Bilinear interpolation is used to make every feature matrix has the same width and height then (2DPCA) is used to get the features. Experimental result shows that (2DPCA) has better performance comparing to traditional (PCA). Minimum distance classification algorithm was used to identify the human gaits. Method was still sensitive to silhouettes segmentation and the matrix alignment method also may lose some useful information.

Oinam Binarani Devi, et al. [8] proposed a (HSV) colour model and robust background subtraction technique using three approaches. a) Frame differencing, the error rate generated by this approach on Wiezmann dataset was $5.5 \%$, Vis or dataset is .2\% and Ut-interaction dataset was $2.7 \%$. b) Simple Gaussian method, the error rate generated by this approach on Wiezmann dataset is $3.2 \%$, Vis or dataset is $2.1 \%$ and Ut-interaction dataset is $2.5 \%$. c) Gaussian Mixture Model, the error rate generated on Wiezmann dataset is $1.5 \%$, Vis or dataset was $1 \%$ and Ut-interaction dataset is $1.7 \%$. Gaussian Mixture Model has lesser error rate in all the dataset chosen and gives a better performance over the above two approaches.

George Bebis, et al. [9] employed a temporal contour projection as gait pattern to extract required feature vectors for classification. Eigenvectors calculated by applying (KPCA) on four gait patterns of each subject yielded a large number of features that can be reduced with Genatics Algorithm (GA). The classification results demonstrated $92.5 \%, 73 \%, 94.5 \%$ and $96.3 \%$ CCR for Silhouette Segmentation and Body Part Labeling (SSBP) algorithm, sensor markers algorithm, active contour model and hough transform and optimal subset of kernel principal component analysis features of spatiotemporal projections of silhouette using GA respectively.

Unlike previous studies this study attempt to identify people from three differences silhouettes gait sequences category which increase the classification performance using simple new approach depends on general shape and gait period.

\section{METHODOLOGY}

Figure 1 shows the flow chart of our approach. The silhouettes sequences were obtained from (TUM-IITKGP) videos then the ROI algorithm was automatically applied to each image. 2D-DWT, edge detection and gait cycle were used as key features. Neural network was used for classification and pattern recognition. The approach is detailed as following.

\subsection{Pre-processing}

ROI analysis is used in many field to reduce data and explore the size and the object boundary, also helping in extract the exact features [10]. Its also used to increase the efficiency of compression for transmittion and storage purposes [11].

Here ROI automatically extracted based on the pixel value. while the white object is targeted and after finding the first correct pixel value, the algorithm compares each pixel with its neighbors to find matching and continues the search and comparis on to determine the largest white space, so that the shapes which have the same pixel value are neglected, finally the whole shape is cropped. Figure 2 shows preprocessing results for several frames, from top to bottom are the original silhouette gait images, ROI cropped silhouette images, respectively. 


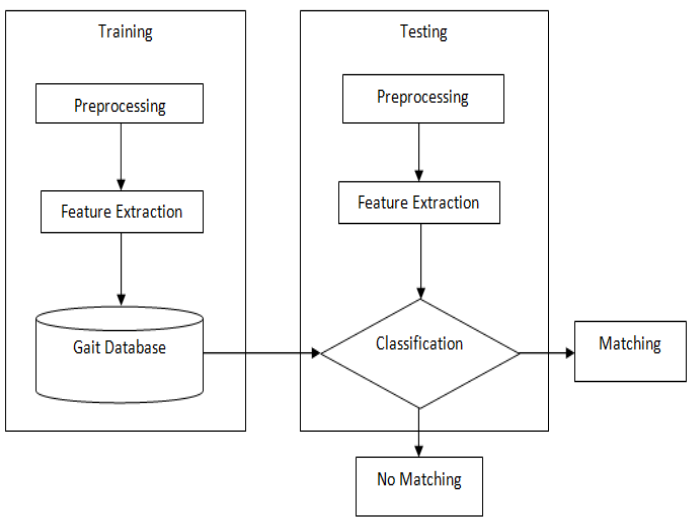

Figure 1. Flow chart of the proposed approach
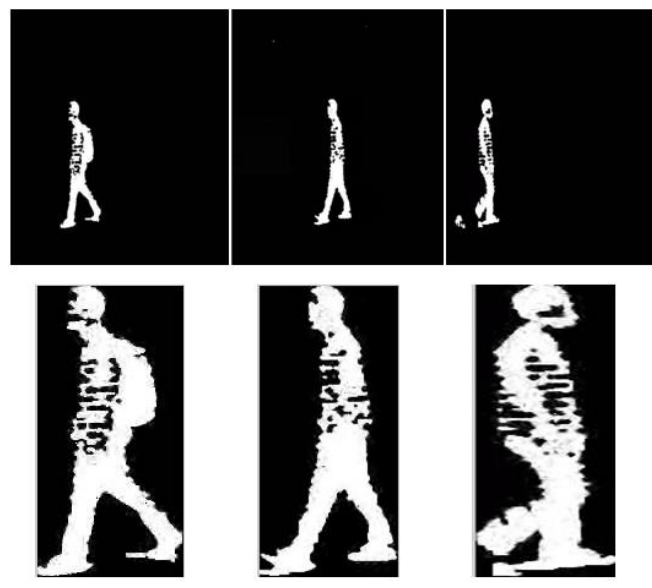

Figure 2. Three frames from three different gait sequence for one subject

\subsection{Features Extraction}

Features have been utilized to identify one class of pattern from another. here features were extracted using discrete wavelet transform, edges and gait cycle.

\subsubsection{Discrete Wavelet Transform}

In general wavelet is a promising set of tools and techniques for multi-scale analysis [12]. Algorithm of this system was using diagonal, horizontal and vertical details coefficient of the three levels of two dimensions discrete wavelet transform which are extracts by 3 levels of low pass filter and high pass filters. Figure 3 shown the 3L-2D-DWT details with ignoring of the approximation results. From top to bottom are the diagonal, horizontal and vertical details coefficient of level one, two and three respectively.
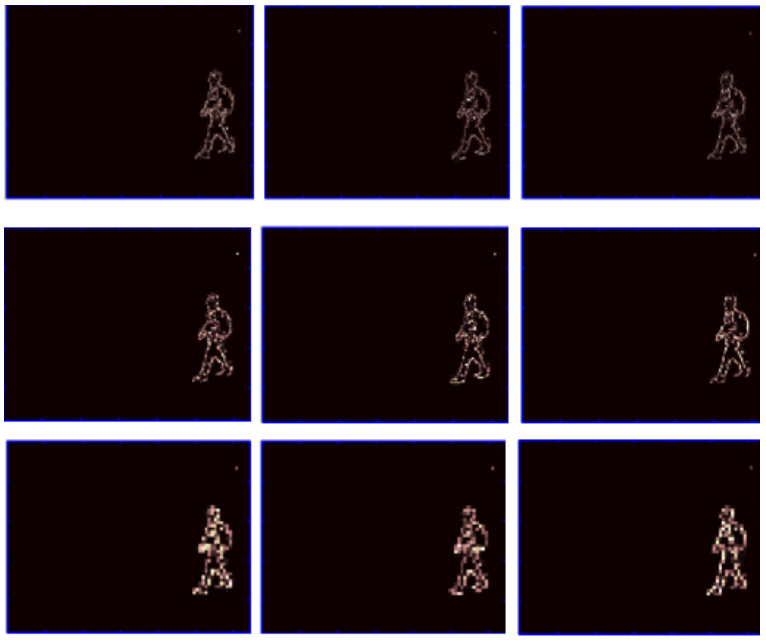

Figure 3. 2D 3Level DWT

\subsubsection{Edge Detection}

It's not a feature by itself but can give the magnitude edge and orientation which allow to extract another feature [13] like general shape. For step size length and cycle length boundary, box technique has been used. In the silhouette a boundary box is created to cover the whole object from outside, the width of this box represents the length of the step size. 


\subsubsection{Gait Cycle}

The act of walking has two basic requisites; periodic movement and ground reaction forces. The full gait cycle start when one foot touch the ground and end when the same foot touch the ground again [14]. There are two main phases in the gait cycle: stance phase (foot in the ground) and swing phase (foot in the air) [15].

Here gait cycle has been estimated from the frames number between the two main phase of gait, by combine the sequance of silhouettes together for each person and the combination area is measured. Then the width of the white shape boundary is extracted which represented the step length. Figure 4 From top to bottom is the first frame from the original silhouette gait images sequence, combined silhouette images, respectively and Figure 5 illustrate sample of gait cycle extracted features and measured length and width.
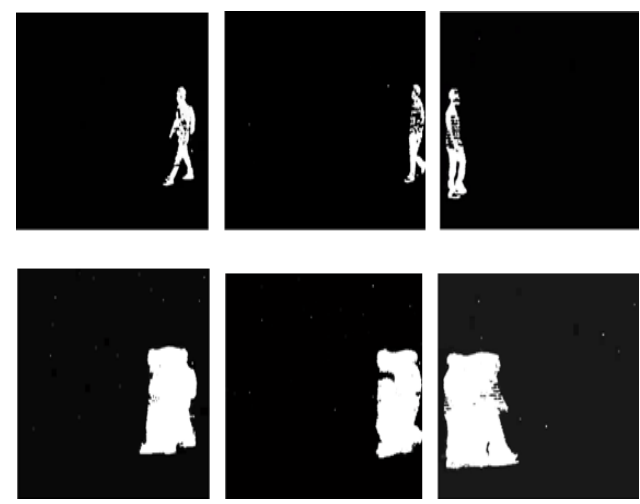

Figure 4. Three frames from three different gait sequence for one subject

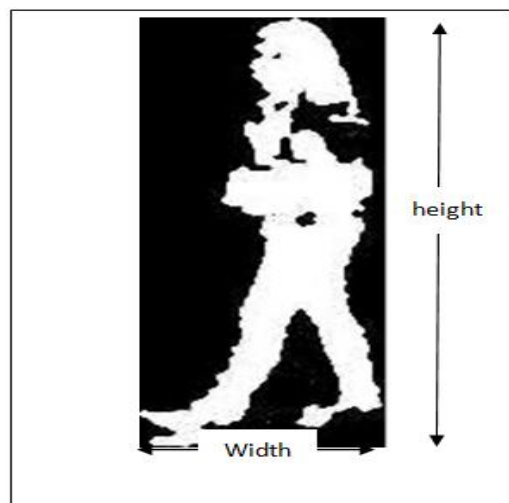

Figure 5. Masured heights and width

\subsection{Classification}

A pattern recognition system mainly have a three steps: one is data acquisition and preprocessing, the other two are feature extraction and decision making [16]. preprocessing convert original information into proper form to be dealt with by computer to perform the mean processes and then recognize or classify. Here a back propagation neural network (BPNN) was used to test and train data that represent the walking person. BPNN is a supervised type of learning depend on modified square mean error algorithm, which aim to reduce the mean square error between the inputs and targets [17].

Simulation are proposed on TUM-IITKGP database of 840 sequences from 35 individuals for each person [18] there are 6 different configurations; 3 configurations (regular walking, walk with hands in pocket and walk with a backpack) have been used, 4 sequence for each of the three configurations. We have trained 15 subjects and each having 120 sequences. For testing 60 sequences were used, as total 3 samples are taken for training and 1 sample for testing for each subject, and 5 subjects were taken as false samples. Figure 6 show training and testing samples.

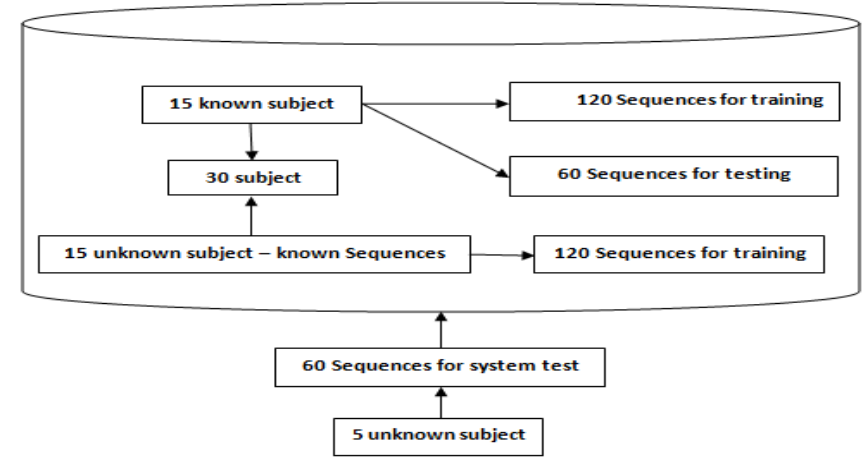

Figure 6. Database samples for neural network training and testing 


\section{RESULTS}

The sensitivity and accuracy of a test can be described as in equastion 1 and equation 2 respectively.

$$
\begin{aligned}
& \text { Sensitivity }=\frac{\mathrm{TP}}{\mathrm{TP}+\mathrm{FN}} \\
& \text { Accuracy }=\frac{\mathrm{TN}+\mathrm{TP}}{\mathrm{TN}+\mathrm{TP}+\mathrm{FN}+\mathrm{FP}}
\end{aligned}
$$

Where: TN; number of correct predictions that an instance is negative.

FP; number of incorrect predictions that an instance is positive.

$\mathrm{FN}$; number of incorrect of predictions that an instance negative.

TP; number of correct predictions that an instance is positive.

All true samples are recognized correctly and the information about actual and predicted classifications done by the system shown in Table 1. The previous studies showed gait recognition techniques based on several algorithms and several dataset; Spatial and Temperal Information (STI), Multi Gait Component (MGC), Sparse Reconstruction based on Metric Learning (SRML), HSV model and Temporal Contour Projection (TCP). HSV model and robust background subtraction technique with visor dataset have the closest result to the Current Study (CA) and MGC also which depends on spatial, temp oral and wavelet. The graph in Figure 7 describe the comparis on between current and previous studies results based on the main studies algorithms accuracy.

Table 1. The Performance Evaluation Parameters

\begin{tabular}{cccc}
\hline \multirow{2}{*}{ Parameters } & \multicolumn{3}{c}{ Configurations } \\
\cline { 2 - 4 } & regular & walk with & walk with \\
walking & hands in pocket & a backpack \\
\hline Accuracy\% & 98.8 & 88 & 95.87 \\
Sensitivity\% & 74 & 100 & 77 \\
\hline
\end{tabular}

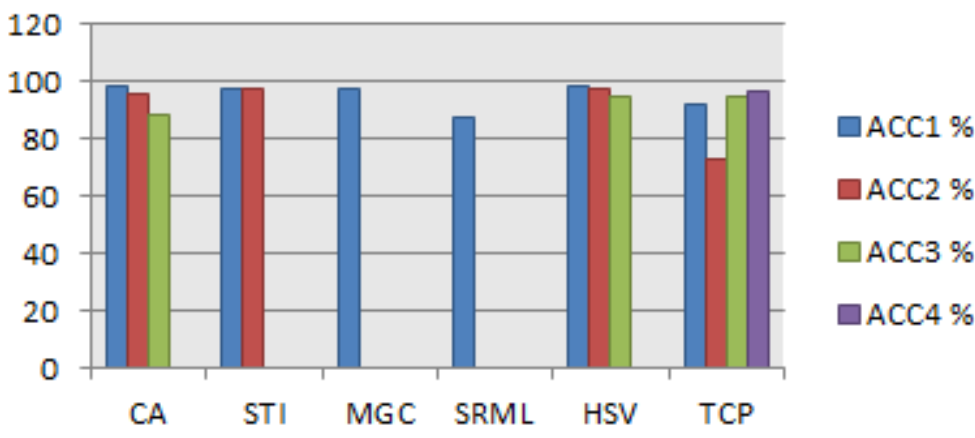

Figure 7. Comparis on between current and previous studies results

Key of (GUI) definition: The screen named interface have three chooses on tool bar :

- Menu; for quit and stay options.

- Help; gave detaieled information about the base and use of whole system.

- About us; for information about programmer and system developer.

The title appear between two images of walking legs as a symbol of gait. Then on the right part of screen three push bottom for selection:

- Upload video; to load data from any folders.

- Search; used for execute the program.

- Share your data with us; used for saved data from external folders.

On the screen left side shown the first frame window which shown the first frame from uploaded video or first image from uploaded images sequence. Also on down right side there is a note about require data and the result appear on the sub screen in the middle under the title (Result) as an (IP) code. When upload video key pressed new window appear to select data (videos or images sequence) immediateltly a data 
selection message appear in new small window to ensure that data was upload successfully then by search key clicked result shown as (IP) code. When exit button selected, the GUI generates an exit message " Are you sure you want to exit" with two choice "No" for keep GUI on and 'Yes" to exit and close the system. Figure 8, Figure 9, Figure 10 and Figure 11 show the GUI, data upload window, uploaded data message and exit message respectively.

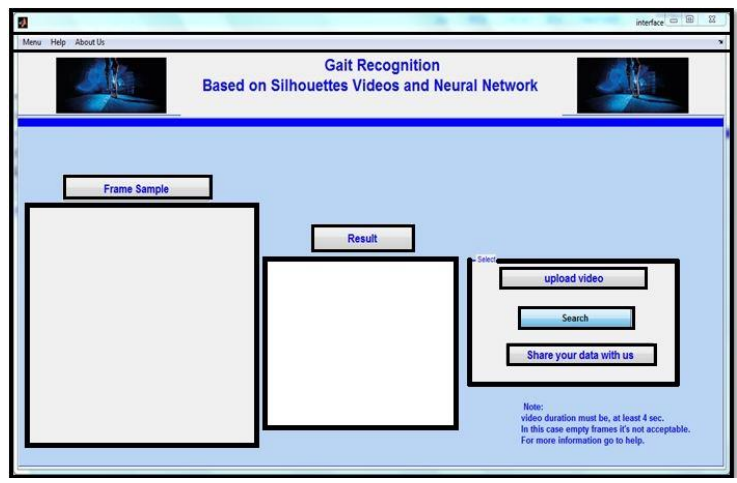

Figure 8. The main graphic user interface of the system

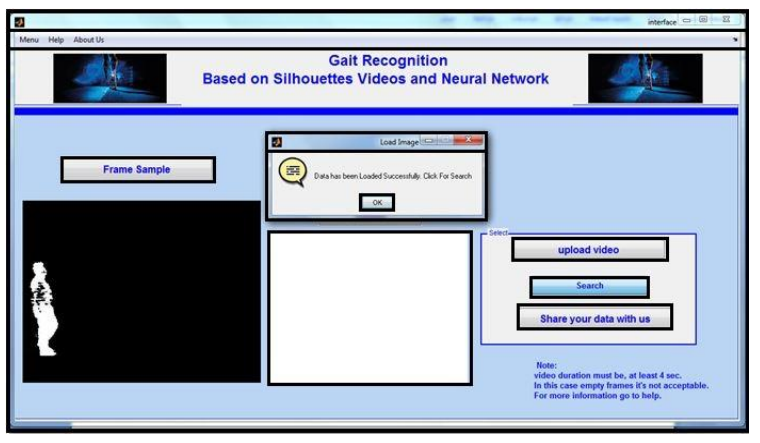

Figure 10. Data uploaded message

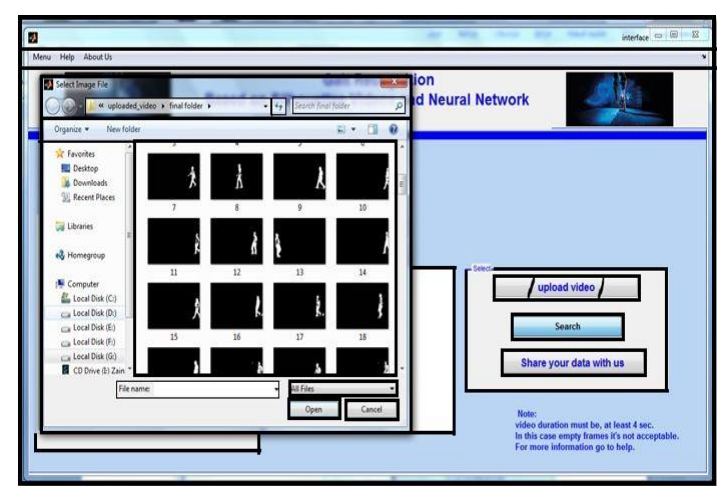

Figure 9. Upload selected video

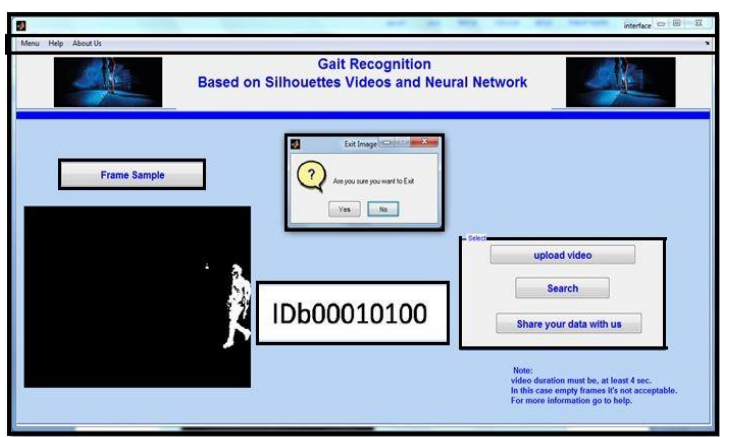

Figure 11. IP code sample with exit message

When the data is loaded, the system be ready to search and display the result. Figure 12 shows a sample of normal walking case and its result as a binary IP. Normal walking has higher accuracy and less sensitivity compared to other cases because of the free hands motion which has the advantages of overall shape and also have a large role in the walking cycle. Another result shown in Figure 13 which illustrate the walking with backpack case. Backpack has a medium accuracy comparied with the other two cases and also like normal walking case have the advantage of hand swinging but it's limited by effects of the backpack load. In Figure 14 the result of walking with hand in the pocket case is displayed. In hand in pocket walking effects of hands motion is not exist which affected the main features of the system by increase the sensitivity of the system compared with two previous cases.

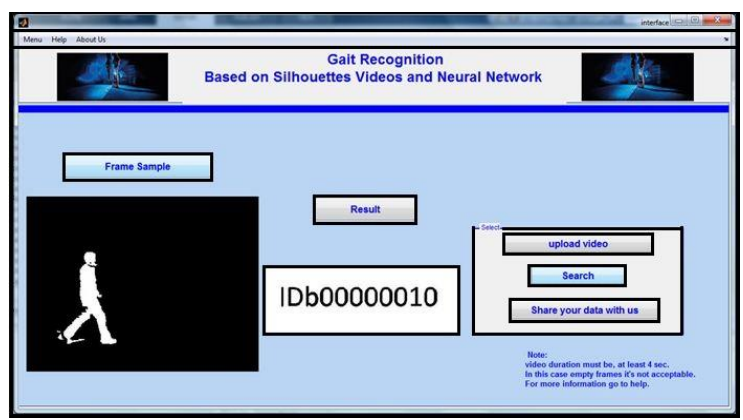

Figure 12. Normal walking

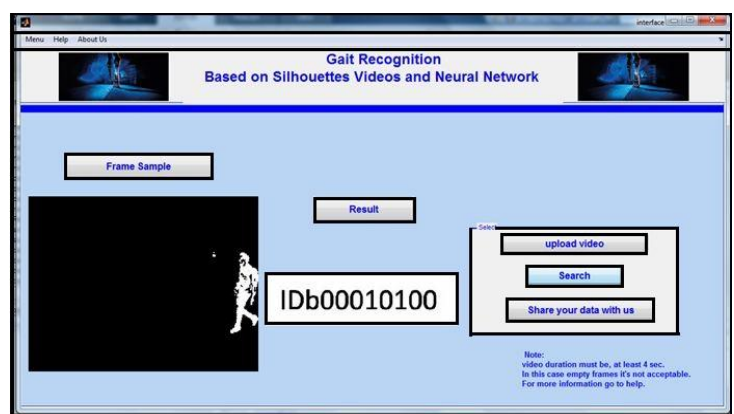

Figure 13. Walking with backpack 


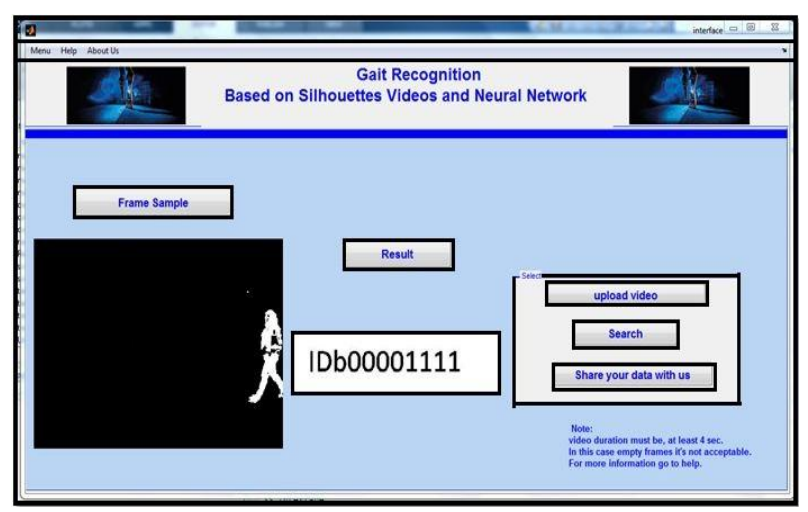

Figure 14. Hand in pocket

\section{CONCLUS ION}

In this paper we have introduced method that based on silhouettes image sequences, (ROI), wavelets and gait cycle for pre-processing and feature extraction and neural networks for recognition human gait. The algorithms are executed on MATLAB (R2014a). TUM-IITKGP database is used with three different configuration of samples sequence. The results show that the total accuracy is $98.8 \%, 95.87 \%$ and $88 \%$ for normal walk, walking with backpack and hand in pocket respectively. In the future its maybe possible to extend the work to include other configurations and more physical activity like fast walking, running and jumping also using current technique with modern convolutional neural networks algorithm to enhance sensitivity.

\section{REFERENCES}

[1] Himanshu Srivastava. A Comparison Based Study on Biometrics for Human Recognition. IOSR Journal of Computer Engineering. 2013; 15(1): 22-29.

[2] V. G. Manjunatha Guru, V. N. Kamalesh. Vision Based Human Gait Recognition System: Observations, Pragmatic Conditions and Datasets. Indian Journal of Science and Technology. 2015; 8(15): 1-15.

[3] Negin K. Hosseini, Md Jan Nordin. Human Gait Recognition: A Silhouette Based Approach. Journal of Automation and Control Engineering. 2013; 1(2): 103-105.

[4] Kohei Arai, Rosa Andrie Asmara. Human Gait Gender Classification in Spatial and Temporal Reasoning. International Journal Advanced Research in Artificial Intelligence. 2012; 1(6): 1-6.

[5] L.R Sudha, Dr. R. Bhavani. Biometric Authorization System using Gait Biometry. International Journal Computer Science, Engineering and Applications. 2011; 1(4): 1-13.

[6] Jiwen Lu, Gang Wang and Pierre Moulin. Human Identity and Gender Recognition From Gait Sequences With Arbitrary Walking Directions. IEEE Transactions on Information Fornsics and Security. 2014; 9(1): 51-61.

[7] Jiny an Chen, Gang Li. Human Gait Identification Based on Difference Image. International Journal Computer. 2014; 9(11): 2744-2751.

[8] Oinam Binarani Devi, Nissi S. Paul, Y. Jay anta Singh. Robust Statistical Approach for Extraction of Moving Human Silhouettes from Videos. International Journal of Information Theory. 2014; 3(3): 55-64.

[9] Faezeh Tafazzoli, George Bebis, Sushil Louis, and Muhammad Hussain. Genetic Feature Selection for Gait Recognition. Electronic Imaging. 2015; 24(1): 013036-1-013036-14.

[10] Rahul Pratap Singh, Parush Agarwal. Extraction of Region of Interest through E-Learning Videos with Matlab. International Journal of Computer Applications. 2013; 68(21): 38-40.

[11] PratikChavada, Narendra Patel and KanuPatel. Region of Interest Based Image Compression. International Journal of Innovative Research in Computer and Communication Engineering. 2014; 2(1): 2320-9798.

[12] Wu Fengxiang. Face Recognition Based on Wavelet Transform and Regional Directional Weighted Local Binary Pattern. Journal of Multimedia. 2014; 9(8): 1017-1023.

[13] B O. Sadiq, S.M. Sani and S. Garba. Edge Detection: A Collection of Pixel based Approach for Colored Images. International Journal of Computer Applications. 2015; 113(5): 29-32.

[14] Magesh. M, Manimaran. S, Murali. M. A Brief on Normal Human Gait Cycle and the Development of Artificial Prosthesis of Human Knee Along With Its Jigs and Fixtures. International Journal of Innovative Research in Science, Engineering and Technology. 2015; 4(2): 132-137.

[15] Agostini V., Balestra G., Knaflitz M. Segmentation and classification of gait cycles. IEEE Tanscations on Neural Systems and Rehabiliation Engineering. 2013; 22(5): 946-952.

[16] Priy anka Sharma and Manavjeet Kaur. Classification in Pattern Recognition: A Review. International Journal of Advanced Research in Computer Science and Software Engineering. 2013; 3(4): 298-306. 
[17] Siddhant C. Joshi and Dr. A.N.Cheeran. MATLAB Based Back-Propagation Neural Network for Automatic Speech Recognition. International Journal of Advanced Research in Electrical, Electronics and Instrumentation Engineering. 2014; 3(7): 10498-10504.

[18] Martin Hofmann, Shamik Sural, Gerhard Rigoll. Gait Recognition in the Presence of Occlusion: A New Dataset and Baseline Algorithms.in Proc. WSCG Communication. 2011: 99-104. 